\title{
SARS-CoV-2 variants and convalescent plasma: reality, fallacies, and opportunities
}

\author{
Arturo Casadevall, Jeffrey P. Henderson, ${ }^{2}$ Michael J. Joyner, ${ }^{3}$ and Liise-anne Pirofski ${ }^{4}$ \\ 'Department of Molecular Microbiology and Immunology, Johns Hopkins School of Public Health, Baltimore, Maryland, USA. ²Departments of Medicine and Molecular Microbiology, Washington University \\ School of Medicine in St. Louis, St. Louis, Missouri, USA. ${ }^{3}$ Department of Anesthesiology and Perioperative Medicine, Mayo Clinic, Rochester, Minnesota, USA. ${ }^{4}$ Departments of Medicine and Microbiology \\ and Immunology, Albert Einstein College of Medicine and Montefiore Medical Center, Bronx, New York, USA.
}

I tion of pandemic severe acute respiratory syndrome coronavirus 2 (SARS-CoV-2) isolates identified viral variants that are less susceptible to neutralization by convalescent plasma (CP), vaccine-elicited plasma/ sera, or SARS- CoV-2 monoclonal antibodies (mAbs) than earlier SARS-CoV-2 strains. The emergence and spread of SARS-CoV-2 variants that evade vaccine immunity would be a cataclysmic development for a world expecting relief from the pandemic and a return to normality, since these could cause new cycles of infection in vaccinated individuals and those who recovered from coronavirus disease 2019 (COVID-19).

Some media reports have suggested that SARS-CoV-2 variants emerged as a consequence of CP use for COVID-19 (1, 2). This hypothesis followed a report that CP treatment in an immunosuppressed patient with COVID-19 was associated with the emergence of new SARS-CoV-2 variant populations in that patient, some of which exhibited reduced susceptibility to antibody neutralization (3). This observation was confirmed in the laboratory, where serial passage of virus in the presence of $\mathrm{CP}$ selected for antibody-resistant SARS-CoV-2 variants, including the $\mathrm{E} 484 \mathrm{~K}$ mutation associated with vaccine resistance (4). These findings established that CP could select for antibody-resistant variants, although this should not be regarded as a surprising result. As a powerful antiviral agent, $\mathrm{CP}$ is expected to exert selective pressure on viral populations in these types of in vitro evolution experiments. In this context, it is notable and reassuring that resistanceassociated viral variants appeared very late, in the eighth passage (4).
RNA viruses exist in quasi-species populations including variants that may predominate under selective pressure from the host's immune system, including both endogenous and passively administered specific antibodies. Specific mAbs may exert pressure that is even more consequential than that of polyclonal preparations such as CP. This is because only a few amino acid changes are necessary to abrogate $\mathrm{mAb}$ binding to a single viral determinant (5). For example, exposure to the respiratory syncytial virus-specific (RSV-specific) mAb palivizumab was associated with the emergence of variant viruses during experimental infection of rats $(6,7)$ as well as during treatment of human infants (8). However, administration of $\mathrm{mAb}$ combinations (oligoclonal "cocktails") can abrogate the emergence of resistant viruses, as demonstrated for SARS-CoV-2 mAbs (9). Nevertheless, the broader array of immune system functions also influences the outcome of host-virus interactions.

Variant drug and/or antibody-resistant viruses are more likely to arise with or without augmentation by specific antibodies (CP or mAbs) in immunocompromised patients, because their generally high viral burdens provide greater opportunity for variant selection. Incidentally, mAbs are theoretically more likely to select for antibody-resistant variants simply because these bind to a single epitope that can be easily abrogated by one or more amino acid changes, whereas CP contains antibodies of multiple specificities. The propensity of resistant variants to emerge in hosts with impaired immunity has been noted with other respiratory viruses such

Conflict of interest: The authors have declared that no conflict of interest exists.

as influenza, for which oseltamivir-resistant influenza viruses were more likely to be identified in immunocompromised individuals (10).

We are now presented with news of globally emergent, antibody-resistant SARS-CoV-2 variants alongside new laboratory evidence that $\mathrm{CP}$ can select for SARS-CoV-2 antibody-resistant variants in immunocompromised individuals. We must be wary of drawing fallacious, causative relationships between these new facts. SARS-CoV-2 variants associated with higher transmissibility emerged during a surge in the United Kingdom (11). During that time, CP use was limited to hospitalized patients who either ultimately died or resolved their infection. For CP to have been responsible for the observed SARS-CoV-2 variant, it would have had to emerge in patients who subsequently transmitted it to the community. This is possible but extremely unlikely within the larger pandemic. A more likely explanation is that variants are emerging from the vast pool of replicating viruses that is presently passaging through hundreds of millions of infected humans (Figure 1). This explanation posits that variants emerge spontaneously during viral replication and that some variants that are resistant to antibody-mediated neutralization are then selected by the developing immune response because they are more fit for survival in immune hosts. In other words, while CP is not the cause of mutations, it can select for resistant variants in immunocompromised hosts who lack normal immune mechanisms that clear virus. Such individuals are under medical care in isolation and thus represent a very unlikely source for the variants circulating in the population. Variants have arisen, and continue to arise (12), amid a backdrop of a lack of 
A

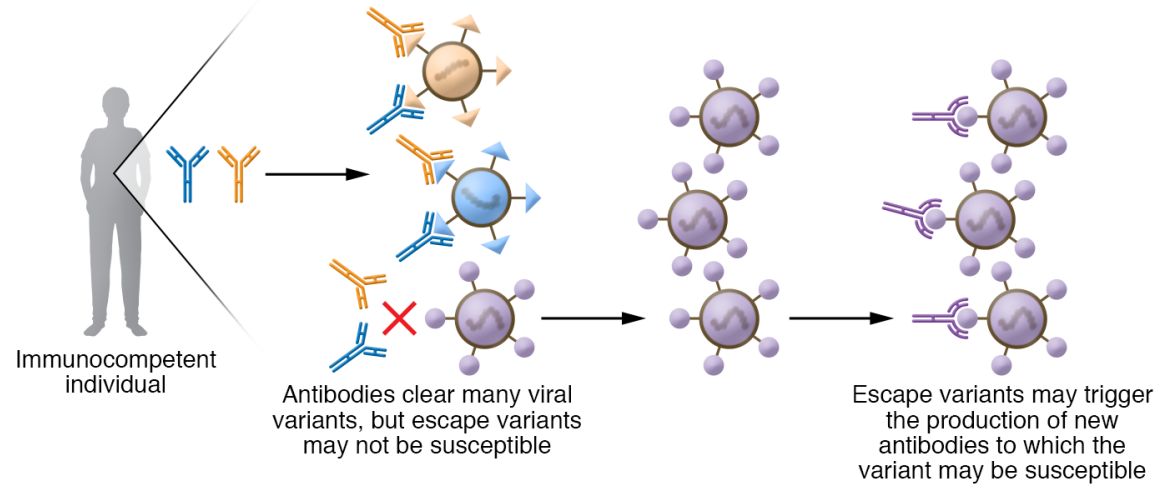

B

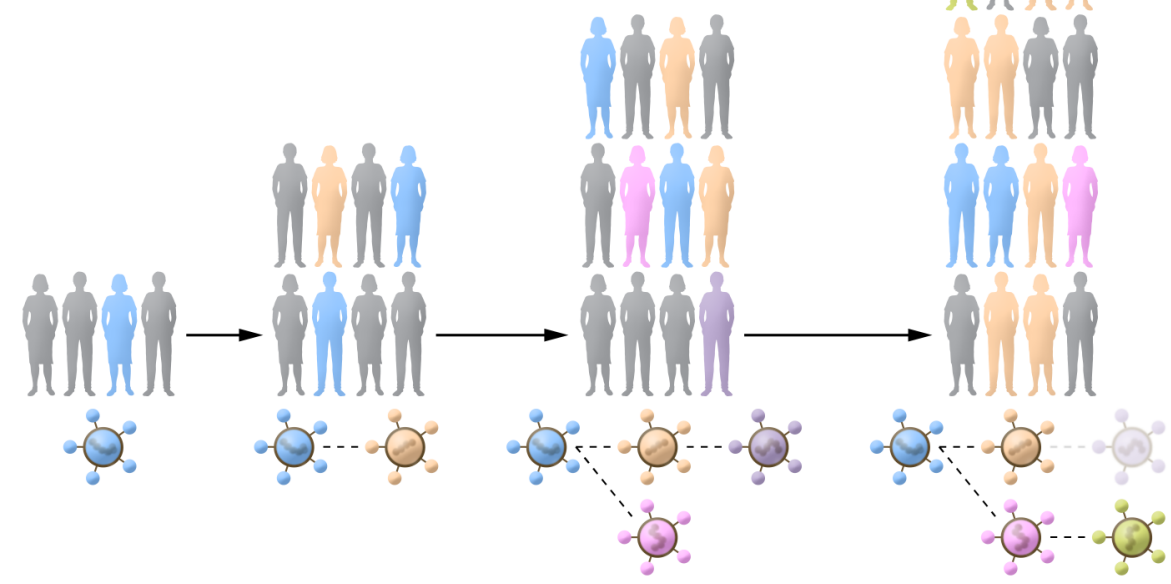

Figure 1. Schema for the emergence of SARS-CoV-2 variants in individuals and populations.

(A) Within an immunocompetent individual, SARS-CoV-2 infection elicits an antibody response to multiple viral determinants. While these antibodies have the capacity to reduce the viral load, ongoing SARS-CoV-2 replication can result in new viral variants that may or may not be susceptible to existing antibodies. In some individuals, especially those with an impaired ability to produce a robust antibody response, viral variants that escape the antibody (and/or other elements of the immune) response may emerge. On the other hand, variants that emerge in an immunocompetent individual can elicit new antibodies to which the variant is susceptible. Thus, plasma containing antibodies produced by such individuals constitutes an immediately available antiviral agent for emerging variants. (B) Viral variants emerge at the population level. New viral variants emerge as a result of chance mutations in individuals who are competent for transmission and replication in populations. Deleterious mutations of the virus are eliminated, while neutral or more fit variants spread in the population when transmission is uninterrupted. While individuals infected with these variants may clear them, widespread transmission will continue when the transmission rate exceeds the rate at which individual immune responses achieve virologic control.

adherence to public health measures such as mask wearing and physical distancing.

Importantly, humans with COVID-19 generate antibodies against the viral species with which they are infected, and these antibodies are found in CP, which is obtained from patients who have recovered from the disease. Notably, endogenous antibodies contribute to SARS-CoV-2 clearance as demonstrated in mAb trials (13). The emergence of resis- tant variants in a population increases in probability with the number of infected hosts. With pandemic spread, particularly in settings where there is insufficient adherence to public health measures such as mask wearing and physical distancing, SARS-CoV-2 is under substantial, population-wide pressure to generate antibody-resistant variants independently of the relatively miniscule CP-treated patient population. The selective pres- sures of community spread are also likely to yield antibody-resistant variants with preserved, or even enhanced, transmission potential relative to those arising from a patient with prolonged viremia. Although the emergence of antibodyresistant variants is a predictable outcome of viral evolution during the ongoing pandemic, there is currently no direct evidence to suggest that circulating variants are a consequence of $\mathrm{CP}$ use.

Some have suggested that $\mathrm{CP}$ use should be avoided in immunocompromised patients, in whom prolonged viremia is possible. However, CP administration has repeatedly been shown to be an effective antiviral agent in this population, and the FDA Emergency Use Authorization revised in February 2021 recognizes these patients (14) as a population that may derive special benefit from CP $(15,16)$. We believe that it is prudent to administer SARS-CoV-2-specific antibodies to these individuals early, in monitored settings, with good infection control practices and adherence to public health measures. Effective COVID-19 therapies are very limited, especially for the viral phase of the disease and should not be withheld because we are concerned about the emergence of antimicrobial resistance.

Only a very small proportion of all individuals who have been infected with SARS-CoV-2 have received $\mathrm{CP}$ or $\mathrm{mAbs}$ for COVID-19 therapy. In contrast, more than 100 million people have been infected with SARS-CoV-2, and the majority of these individuals have made antibodies similar to those found in $\mathrm{CP}$ or $\mathrm{mAb}$ preparations. Among these are neutralizing antibodies that put selective pressure on infecting virus to evade antibody-mediated neutralization and maintain a replicating population within the host. Each person who is infected and mounts an antibody response has a low probability of selecting for a resistant variant. However, when that low probability is multiplied by the hundreds of millions of people who have been infected, the likelihood of variant emergence is a near certainty. Occam's razor favors the simplest explanation, which is that antibody-resistant variants emerged spontaneously, were selected by natural immune responses in infected individuals, and are spreading because they offer a fitness 
advantage in a human population that is increasingly immune to the founder SARSCoV-2 strains.

Within these emerging circumstances, important new opportunities for CP use arise. Although SARS-CoV-2 variants may elude antibodies elicited by earlier, ancestral SARS-CoV-2 strains, individuals who recover from variant COVID-19 are likely to generate $\mathrm{CP}$ capable of neutralizing variants. Hence, variant CP could be a potential antidote for variant SARS-CoV-2. As variant SARS-CoV-2 strains spread in human populations, recently donated CP units will be especially efficacious for patients with variant COVID-19. Thus, strategies to deploy CP could be tailored to align with currently circulating virus strains. This provides an additional rationale for surveillance of virus strains, which is necessary to follow SARSCoV-2 evolution, just as it is for influenza. As we move toward a hopeful, but increasingly uncertain, second quarter of 2021, every effort should be made to conduct real-time viral surveillance with increased sequencing capacity designed to identify the emergence, type, and prevalence of variant SARS-CoV-2 strains. The resulting knowledge should inform CP characterization by determining each unit's ability to neutralize relevant circulating viral variants. Going forward, matching CP characteristics to each patient's virus type may become a useful precision medicine approach at the front lines of the pandemic.

\section{Acknowledgments}

AC is supported in part by the $\mathrm{NIH}$ (AI052733, AI15207, and HL059842). JH is supported in part by the NIH (DK111930) and The Longer Life Foundation. MJJ is supported in part by the National Heart, Lung, and Blood Institute (NHLBI), NIH (5R35HL139854). LP is funded in part by the Mathers Foundation, the NIH (R01AI123654 and R01AI143453), and the National Center for Advancing Translational Sciences (NCATS), NIH (3UL1TR002556-04S1).

Address correspondence to: Arturo Casadevall, Department of Molecular Microbiology and Immunology, Johns Hopkins School of Public Health, 615 N. Wolfe Street, Room E5132, Baltimore, Maryland 21205, USA. Phone: 410.955.3457; Email: acasadevall@jhu.edu.

1. Cortez MF. Chronic Covid-19 and convalescent plasma may boost mutation risk. Bloomberg. February 6, 2021. Accessed February 24, 2021 https://www.bloombergquint.com/onweb/ chronic-covid-19-and-convalescent-plasmamay-boost-mutation-risk.

2. Walker M. Did convalescent plasma for COVID19 unleash viral mutation? MedpageToday. February 5, 2021. Accessed February 24, 2021. https://www.medpagetoday.com/infectiousdisease/covid19/91088.

3. Kemp SA, et al. SARS-CoV-2 evolution during treatment of chronic infection [published online February 5, 2021]. Nature. https://doi. org/10.1038/s41586-021-03291-y.

4. Andreano E, et al. SARS-CoV-2 escape in vitro from a highly neutralizing COVID-19 convalescent plasma [preprint]. https://doi. org/10.1101/2020.12.28.424451. Posted on on bioRxiv December 28, 2020.

5. Yurkovetskiy L, et al. Structural and functional analysis of the D614G SARS-CoV-2 spike protein variant. Cell. 2020;183(2):608-751.

6. Zhao X, et al. Respiratory syncytial virus escape mutant derived in vitro resists palivizumab prophylaxis in cotton rats. Virology. 2004;318(2):608-612.

7. Zhao X, Sullender WM. In vivo selection of respiratory syncytial viruses resistant to palivizumab. J Virol. 2005;79(2):185-3968.

8. Adams O, et al. Palivizumab-resistant human respiratory syncytial virus infection in infancy. Clin Infect Dis. 2010;51(2):185-188.

9. Baum A, et al. Antibody cocktail to SARSCoV-2 spike protein prevents rapid mutational escape seen with individual antibodies. Science. 2020;369(2):361-1018.

10. Kossyvakis A, et al. Antiviral susceptibility profile of influenza A viruses; keep an eye on immunocompromised patients under prolonged treatment. Eur J Clin Microbiol Infect Dis. 2017;36(2):361-371.

11. World Health Orginization. SARS-CoV-2 Variant - United Kingdom of Great Britain and Northern Ireland. WHO; 2020. Accessed February 24, 2021. https://www.who.int/csr/don/21-december2020-sars-cov2-variant-united-kingdom/en/.

12. Mascola JR, et al. SARS-CoV-2 viral variants-tackling a moving target [published on February 11, 2021]. Jama. https://doi.org/10.1001/ jama.2021.2088.

13. Weinreich DM, et al. REGN-COV2, a neutralizing antibody cocktail, in outpatients with Covid19. N Engl J Med. 2021;384(3):238-251.

14. Food and Drug Administration. Investigational COVID-19 Convalescent Plasma. Guidance for Industry. FDA; 2021. Accessed February 24, 2021. https://www.fda.gov/regulatory-information/search-fda-guidance-documents/investigational-covid-19-convalescent-plasma.

15. Senefeld JW, et al. Therapeutic use of convalescent plasma in COVID-19 patients with immunodeficiency [preprint]. https://doi.org/10.11 01/2020.11.08.20224790. Posted on medRxiv November 10, 2020.

16. Thompson MA, et al. Convalescent plasma and improved survival in patients with hematologic malignancies and COVID-19 [preprint]. https:// doi.org/10.1101/2021.02.05.21250953. Posted on medRxiv February 05, 2021. 